
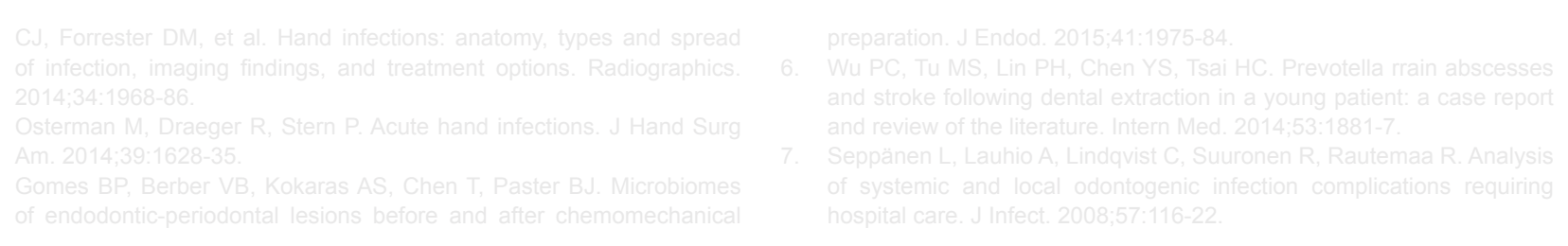

\title{
Enfisema Subcutâneo e Pneumomediastino Durante uma Extração Dentária
}

\section{Subcutaneous Emphysema and Pneumomediastinum During a Tooth Extraction}

André FERREIRA SIMÕES $\square^{1}$, Joana Barbosa RODRIGUES², Sara ÚRIA², Glória NUNES DA SILVA²

Acta Med Port 2018 Jul-Aug;31(7-8):435-439 - https://doi.org/10.20344/amp.8431

RESUMO

O enfisema subcutâneo cervicofacial e mediastínico é uma possível complicação, rara, após procedimentos dentários. Deve-se distinguir de outras situações como o hematoma, reação alérgica ou angioedema e infeção. Descreve-se o caso de uma puérpera de 20 anos com múltiplas cáries dentárias, que desenvolveu enfisema subcutâneo cervicofacial, complicado de pneumomediastino, após extração incompleta do segundo molar inferior direito. O diagnóstico da complicação foi clínico e imagiológico e a situação resolveu sob vigilância, com antibioterapia, em internamento. O caso pretende chamar a atenção para a necessidade de se diagnosticar e tratar precocemente esta complicação, devido ao risco de compromisso da via aérea, embolia gasosa, infeção, sépsis e morte.

Palavras-chave: Enfisema Mediastínico; Enfisema Subcutâneo; Extração Dentária

\section{ABSTRACT}

Subcutaneous emphysema is a possible but rare complication after dental procedures. The condition should be distinguished from other situations, such as hematoma, allergic reaction or angioedema, and infection. We describe the case of a 20-year-old puerperal woman, with multiple dental caries, who developed cervicofacial subcutaneous emphysema complicated by pneumomediastinum, following an incomplete extraction of the lower right second molar. This was diagnosed clinically and through imaging tests, and the situation resolved after hospital admission, with antibiotics and close monitoring. The case underlines the need to diagnose and treat this complication early, because of the risk of airway compromise, air embolism, infection, sepsis and death.

Keywords: Mediastinal Emphysema; Subcutaneous Emphysema; Tooth Extraction

\section{INTRODUÇÃO}

O enfisema subcutâneo cervicofacial caracteriza-se pela presença de ar dentro dos tecidos moles nestas regiões, é uma complicação pouco frequente da extração dentária, e foi descrito pela primeira vez por Turnbull em 1900. ${ }^{1}$ A sua extensão para o mediastino é rara e potencialmente fatal, mas tem sido relatada crescentemente devido à diversificação de técnicas e procedimentos dentários. ${ }^{2,3}$

Este artigo relata o caso de uma mulher de 20 anos com enfisema subcutâneo cervicofacial e pneumomediastino, como complicação de uma extração dentária. O diagnóstico foi possível pela suspeição clínica e confirmado nos exames de imagem. A evolução foi favorável com tratamento conservador.

\section{CASO CLÍNICO}

Mulher de 20 anos, puérpera há um mês, com múltiplas cáries dentárias, sem outros antecedentes pessoais relevantes e sem medicação habitual. Foi submetida a intervenção estomatológica, com intenção de extração do segundo molar inferior direito. Realizou anestesia local infiltrativa com cloridrato de articaína a 4\% com epinefrina a 1:200 000. Durante o procedimento cirúrgico iniciou queixas de edema da face, crepitação subcutânea e dor torácica, pelo que foi encaminhada para o serviço de urgência.

À observação no serviço de urgência apresentava enfisema subcutâneo da hemiface direita, com envolvimento periorbitário e extensão bilateral para a região cervical até à região supraclavicular e à região esternal. Não apresentava queixas nem sinais de dificuldade respiratória, nem instabilidade hemodinâmica ou elétrica. Não tinha alterações relevantes na avaliação analítica. O eletrocardiograma estava normal e os marcadores de isquémia miocárdica seriados foram negativos. A radiografia do tórax e do pescoço mostraram a presença de ar subcutâneo localizado às regiões cervical, supraclavicular e mediastínica (Fig.s 1 e 2). A tomografia computorizada (TC) cervicofacial e torácica revelava enfisema da hemiface direita, com compromisso assimétrico do espaço dos mastigadores, de predomínio direito, condicionando assimetria e redução do diâmetro transverso da coluna aérea da orofaringe (Fig. 3).

\footnotetext{
1. Serviço de Medicina Intensiva. Hospital de Vila Franca de Xira. Vila Franca de Xira. Portugal.

2. Serviço de Medicina Interna III. Centro Hospitalar Lisboa Norte. Lisboa. Portugal.

$\triangle$ Autor correspondente: André Ferreira Simões. andrevicentesimoes@gmail.com

Recebido: 04 de novembro de 2016 - Aceite: 22 de maio de 2018| Copyright @ Ordem dos Médicos 2018
} 


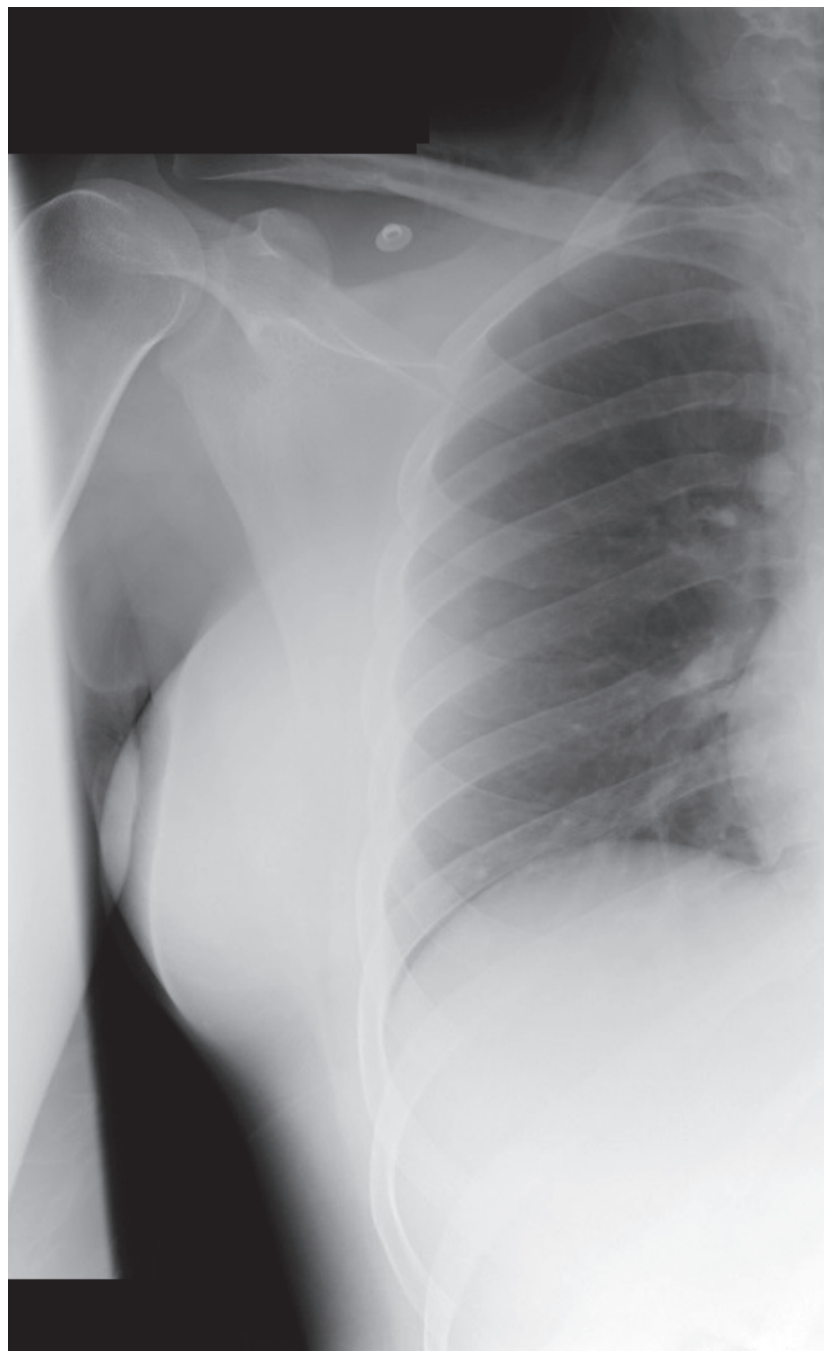

Figura 1 - Radiografia torácica mostrando enfisema mediastínico

O enfisema estendia-se aos compartimentos cervicais de forma assimétrica e ao mediastino anterior, médio e posterior (Fig.s 4 e 5). O exame mostrava ainda as múltiplas peças dentárias com evidência de cáries.

Durante o internamento referiu dor ligeira retrosternal e torácica esquerda, interpretada no contexto do pneumomediastino e da tensão mamária. A dor cedia com paracetamol oral e após a extração do leite com bomba de sucção. Manteve repouso, monitorização cardiorrespiratória e iniciou antibioterapia profilática endovenosa, que cumpriu durante 14 dias, com amoxicilina $1 \mathrm{~g}$ de $8 / 8$ horas, e metronidazol $500 \mathrm{mg} \mathrm{8/8}$ horas. Durante o internamento procedeu-se a extração de raiz inclusa e de um quisto radicular. No início do internamento verificou-se uma elevação dos parâmetros inflamatórios: leucócitos $13,470 \times 10^{9} / \mathrm{L}$ e proteína C reativa $1,53 \mathrm{mg} / \mathrm{dL}$, com normalização ao longo do internamento.

Na reavaliação imagiológica, por TC cervical e torácica, verificou-se diminuição significativa do enfisema cervicofacial e do mediastino (Fig.s 6 e 7). Teve alta, assintomática, após nove dias de internamento. Na reavaliação após quinze dias mostrou ausência de sinais de enfisema subcutâneo, e reabsorção total do pneumomediastino na radiografia torácica (Fig. 8).

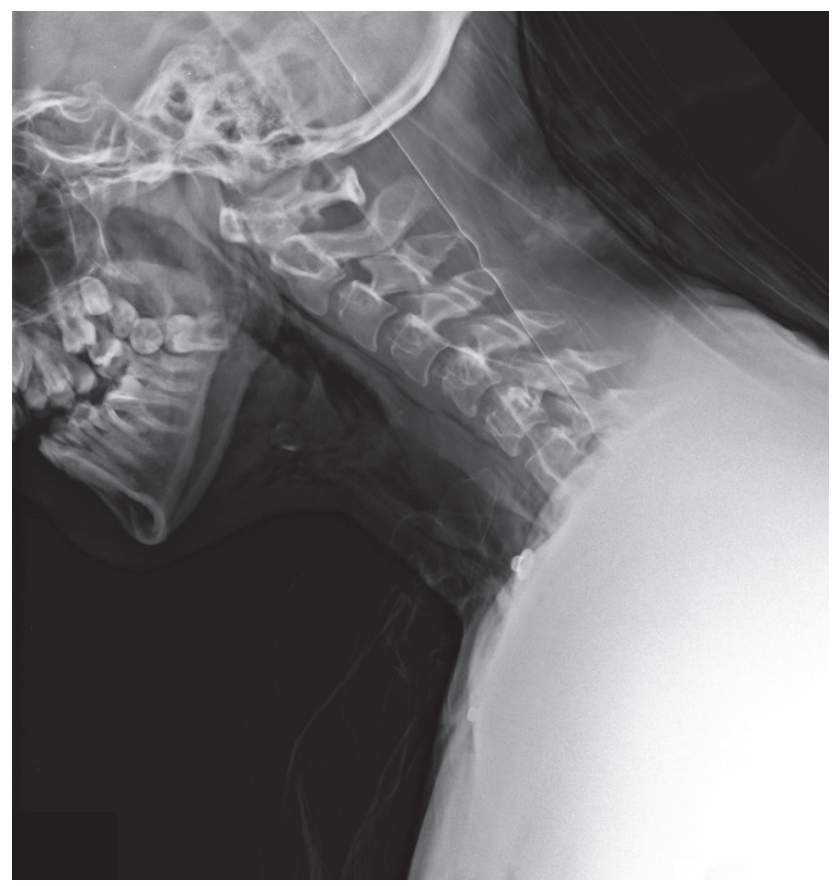

Figura 2 - Radiografia cervical mostrando a presença de ar cervico-facial 


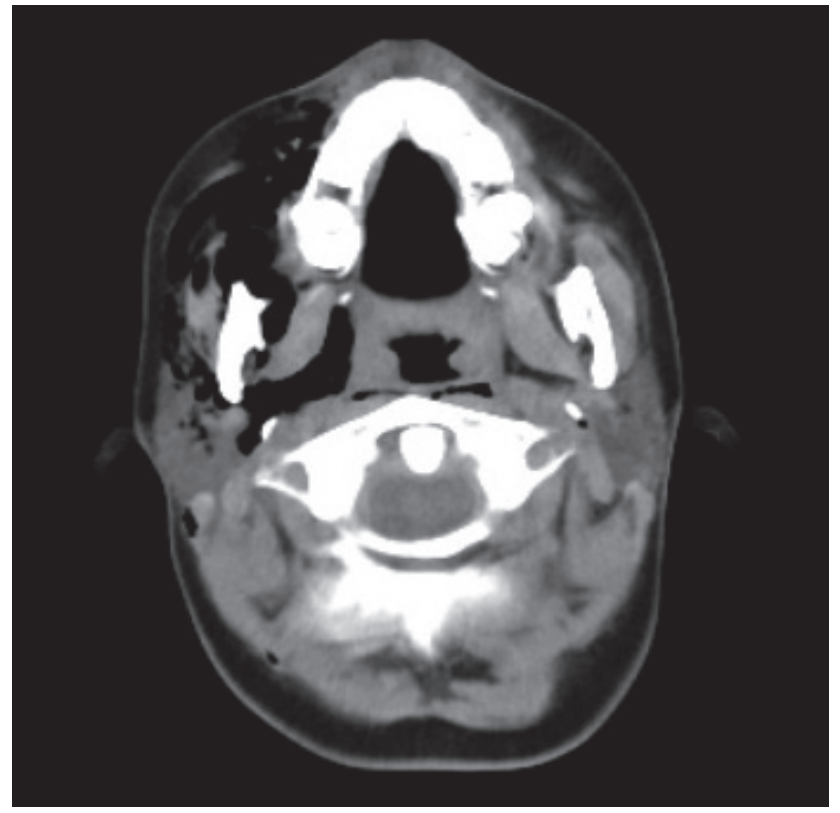

Figura 3 - Tomografia computorizada mostrando enfisema da hemiface direita, com compromisso assimétrico do espaço dos mastigadores e redução do diâmetro transverso da coluna aérea da orofaringe

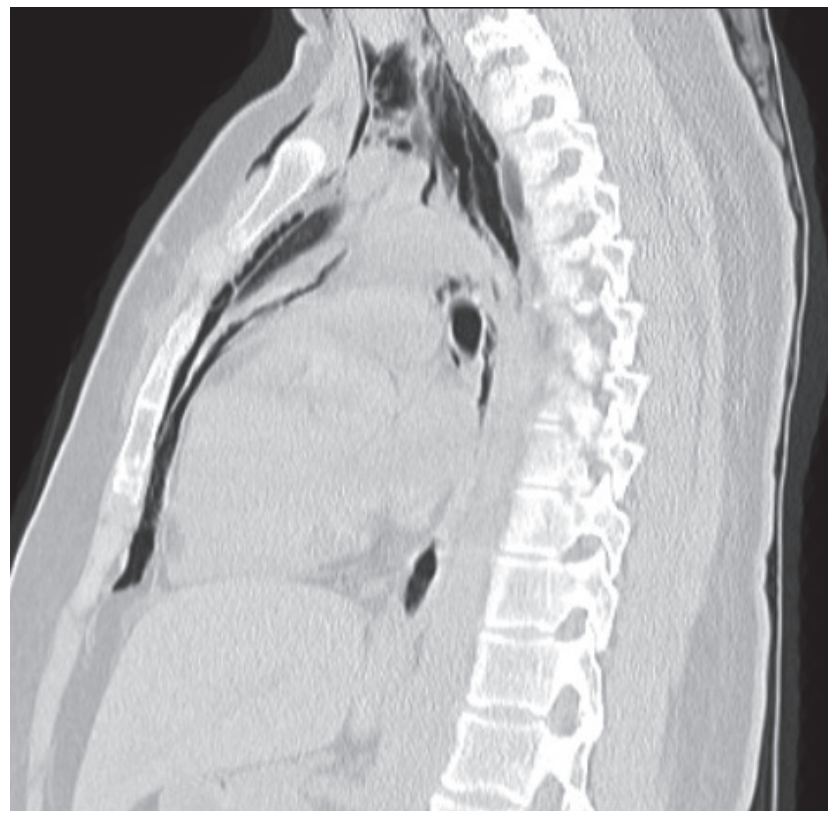

Figura 4 - Tomografia computorizada mostrando presença de ar subcutâneo localizado às regiões cervical, supraclavicular e mediastínica

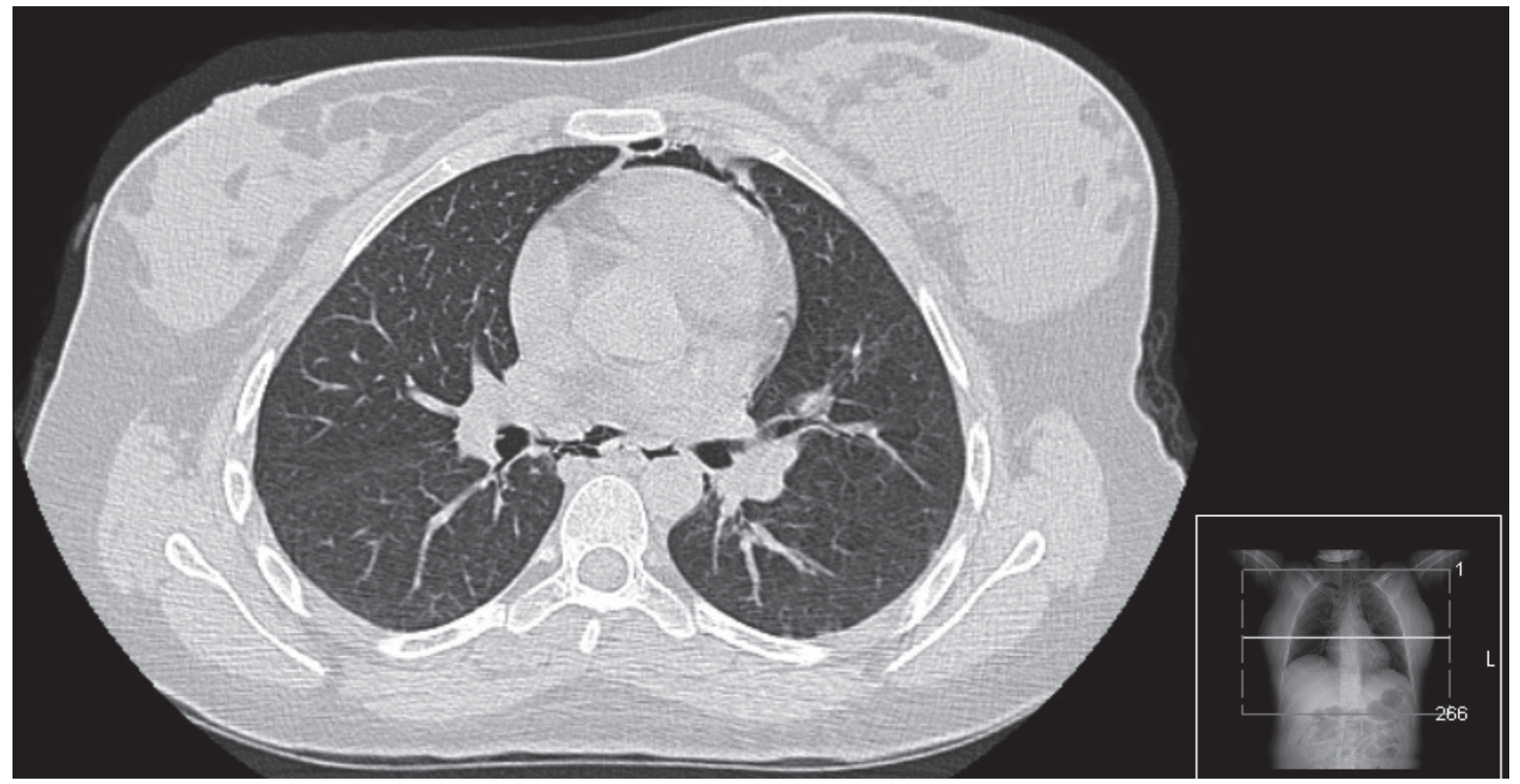

Figura 5 - Tomografia computorizada mostrando presença de ar mediastínico

\section{DISCUSSÃO}

O enfisema é uma complicação rara da extração dentária. As bases das raízes dos três molares inferiores comunicam com os espaços sublinguais e submandibulares. Estes espaços comunicam, por sua vez, com os espaços pterigomandibular, parafaríngeo e retrofaríngeo, sendo através deste último que o ar se pode estender para o mediastino e para o espaço pleural.4,5 $\mathrm{O}$ espectro de manifestações clínicas do enfisema pode ir desde o edema local e a crepitação à palpação superficial, ${ }^{6}$ até à disfagia, disfonia, dispneia e toracalgia. ${ }^{7}$ A evolução para um pneumotórax ou pneumo- pericárdio é excecional. ${ }^{8}$

O enfisema cervico-facial pode resultar de vários procedimentos odontológicos, mesmo quando são realizados corretamente. A utilização de broca pneumática de alta velocidade ou dispositivos com seringa de ar ou spray e soluções para o sistema de canais (particularmente peróxido de hidrogénio) estão associados mais frequentemente à complicação. ${ }^{9,10}$

O diagnóstico é clínico e imagiológico e deve ser feito o diagnóstico diferencial com hematoma, reação alérgica, angioedema ou infeção. ${ }^{11}$ No caso apresentado, o edema 


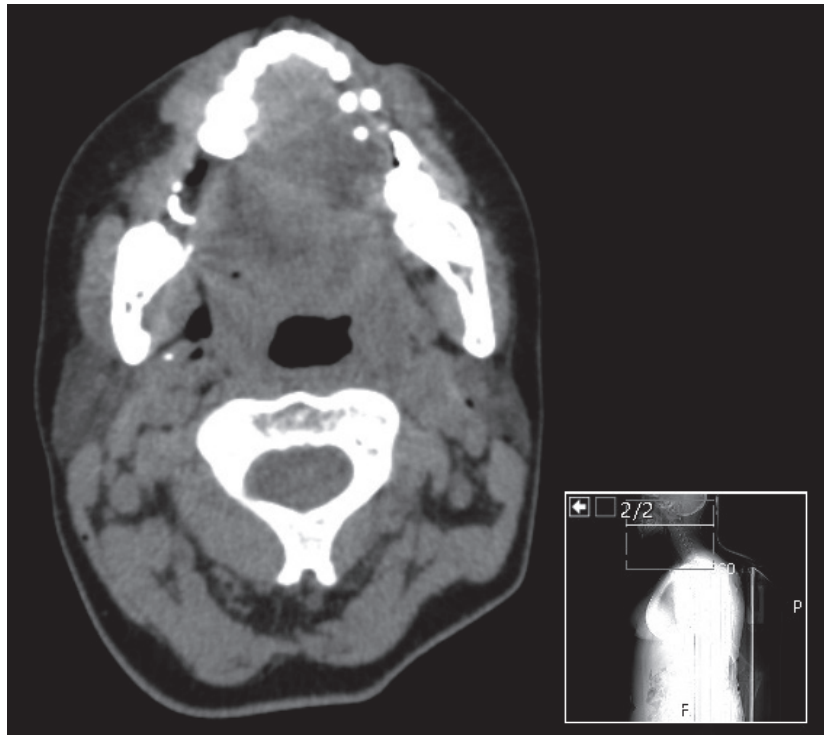

Figura 6 - Tomografia computorizada mostrando resolução do enfisema da hemiface direita e cervical

da face e enfisema subcutâneo manifestaram-se antes da conclusão da extração dentária. Descartou-se a possibilidade de a dor torácica ter outra etiologia através dos marcadores de isquémia miocárdica, do eletrocardiograma e do ecocardiograma, que foram negativos. A tomografia cervicofacial e torácica confirmou o diagnóstico e a extensão da complicação.

No caso descrito optou-se por uma estratégia de tratamento conservador, mantendo a doente internada, em vigilância e sob antibioterapia dupla, com amoxicilina e metronidazol. Estes foram escolhidos para cobrir a flora oral, nomeadamente os microrganismos anaeróbios, atendendo aos múltiplos focos sépticos dentários identificados e à extensão do enfisema às regiões profundas cervicofaciais e ao mediastino. Durante o internamento foi realizada extração de raiz inclusa. Apesar destas complicações poderem determinar compromisso da via aérea, embolia gasosa, infeção, sépsis e morte, o caso por nós relatado foi prontamente diagnosticado e tratado, e evoluiu sem intercorrências ou quaisquer sequelas.

\section{PROTECÇÃO DE PESSOAS E ANIMAIS}

Os autores declaram que os procedimentos seguidos estavam de acordo com os regulamentos estabelecidos pelos responsáveis da Comissão de Investigação Clínica e Ética e de acordo com a Declaração de Helsínquia da Associação Médica Mundial.

\section{CONFIDENCIALIDADE DOS DADOS}

Os autores declaram ter seguido os protocolos do seu centro de trabalho acerca da publicação de dados.

\section{CONSENTIMENTO DO DOENTE}

Obtido.

\section{CONFLITOS DE INTERESSE}

Os autores declaram não terem qualquer conflito de interesse relativamente ao presente artigo.

\section{FONTES DE FINANCIAMENTO}

Os autores declaram não ter recebido subsídios ou bolsas para a elaboração do artigo.

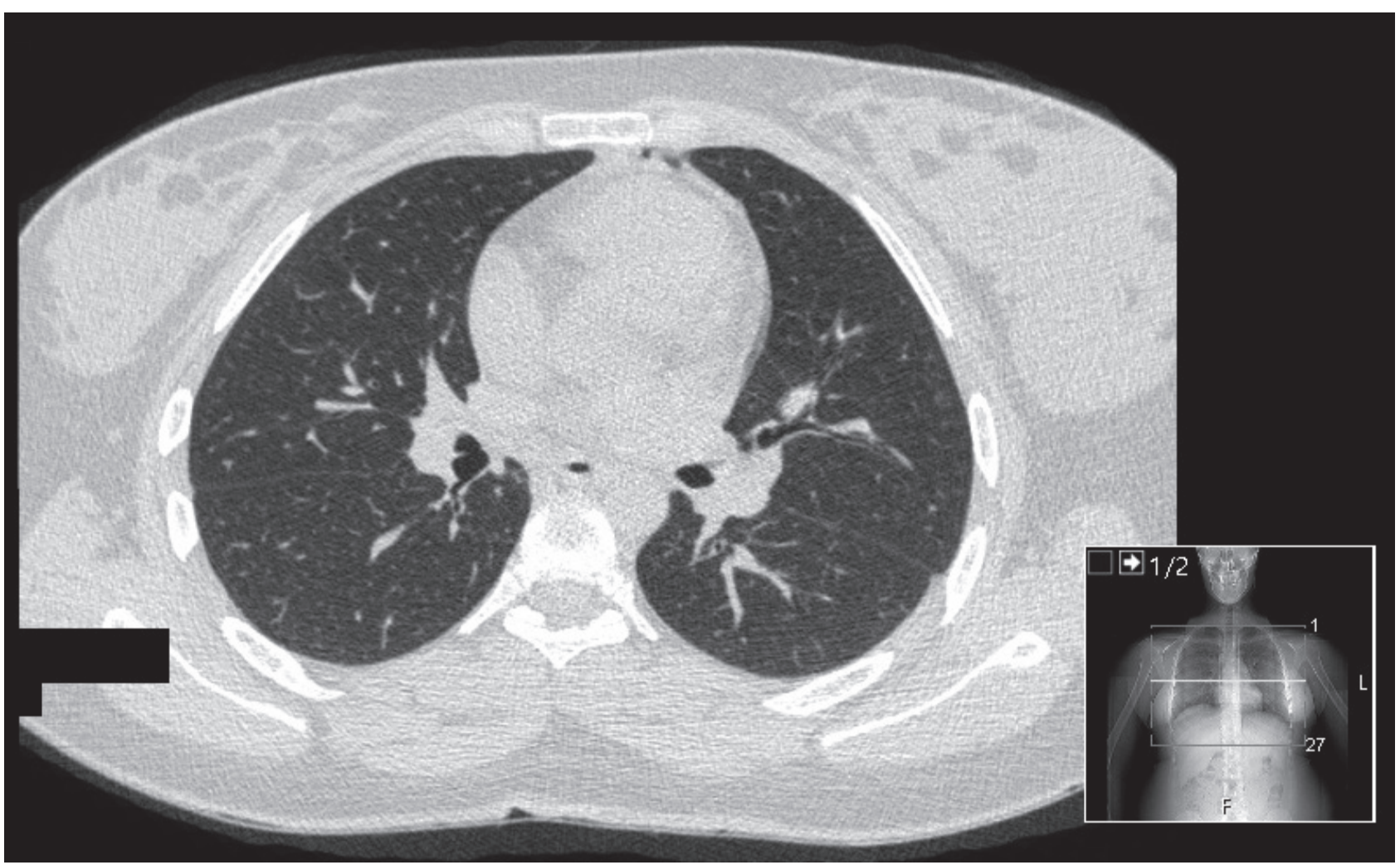

Figura 7 - Tomografia computorizada mostrando resolução do enfisema mediastínico 


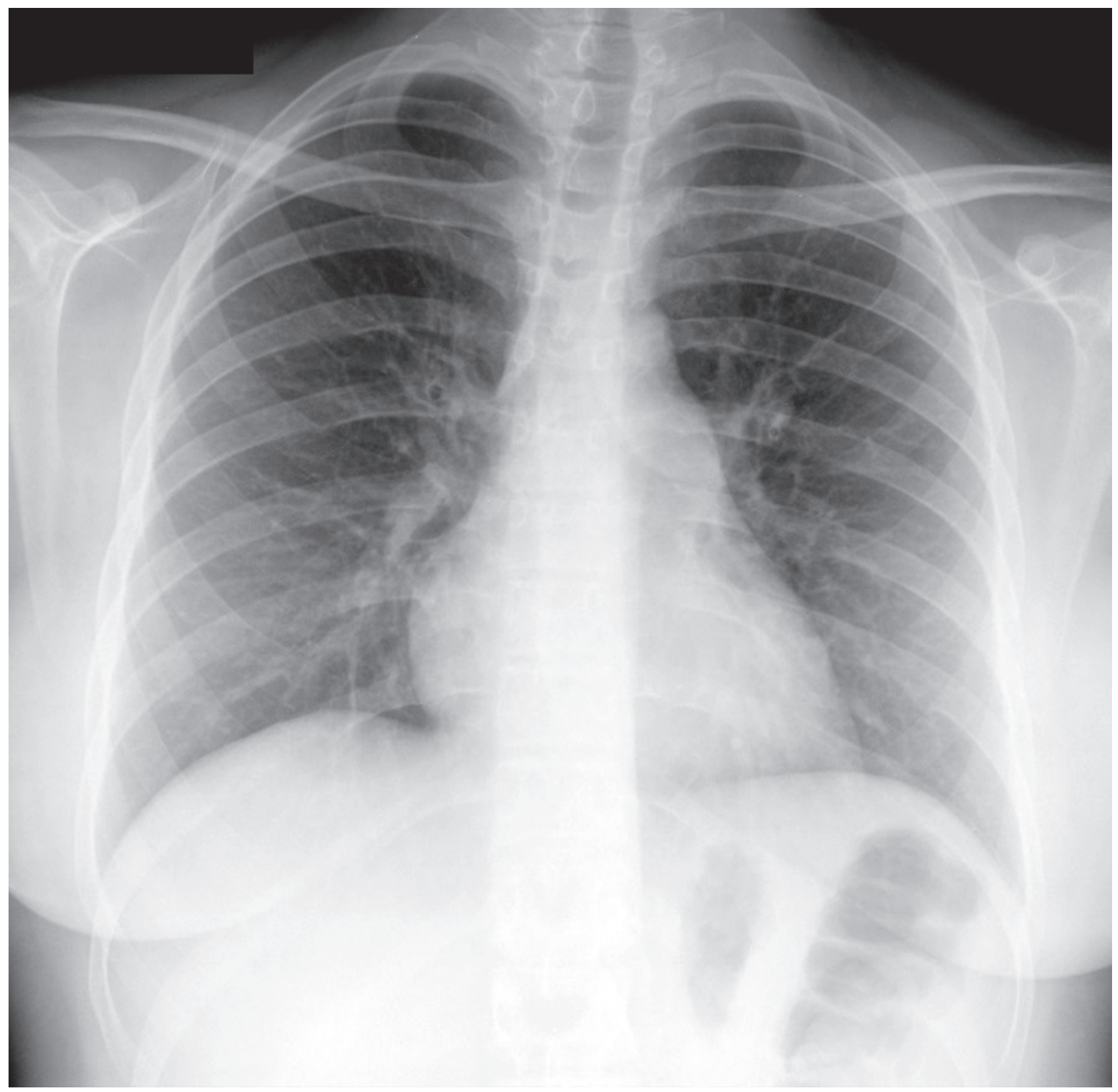

Figure 8 - Radiografia torácica após resolução do pneumomediastino

\section{REFERÊNCIAS}

1. Turnbull A. A remarkable coincidence in dental surgery. $\mathrm{Br}$ Med J. 1900;1:1131.

2. Pousios D, Panagiotopoulos N, Sioutis N, Piyis A, Gourgiotis S. latrogenic pneumomediastinum and facial emphysema after surgical tooth extraction. Ann Thorac Surg. 2010;89:640.

3. Chen SC, Lin FY, Chang KJ. Subcutaneous emphysema and pneumomediastinum after dental extraction. Am J Emerg Med. 1999;17:678-80.

4. Arai I, Aoki T, Yamazaki H, Ota Y, Kaneko A. Pneumomediastinum and subcutaneous emphysema after dental extraction detected incidentally by regular medical checkup: a case report. Oral Surg Oral Med Oral Pathol Oral Radiol Endod. 2009;107:e33-8.

5. Chena $\mathrm{CH}$, Chang $\mathrm{H}$, Liua HC, Hung TT, Huang W. Pneumothorax, pneumomediastinum and pneumopericardium complications arising from a case of wisdom tooth extraction. Rev Port Pneumol. 2012;18:1947 .
6. Yang SC, Chiu TH, Lin TJ, Chan HM. Subcutaneous emphysema and pneumomediastinum secondary to dental extraction: a case report and literature review. Kaohsiung J MedSci. 2006;22:641-5.

7. Cardo VA Jr, Mooney JW, Startigos GT. latrogenic dental air emphysema: report of a case. J Am Dent Assoc. 1972;85:144-7.

8. Salib RJ, Valentine DL, Atchtar S. Surgical emphysema following dental treatment. J Laryngol Otol. 1999;133:756-8.

9. Noble WH. Mediastinal emphysema resulting from extraction of an impacted mandibular third molar. J Am Dent Assoc. 1972;84:368-70.

10. Heyman SN, Babayof I. Emphysematous complications in dentistry, 1960-1993: an illustrative case and review of the literature. Quintessence Int. 1995;26:535-43.

11. Barkdull TJ. Pneumothorax during dental care. J Am Board Fam Med. 2003;16:165-9.

12. Davies DE. Pneumomediastinum after dental surgery. Anaesth Intensive Care. 2001;29:638-41 